\title{
MOLECULAR HYDROGEN EMISSION FROM
}

\section{ULTRALUMINOUS INFRARED GALAXIES}

\author{
P.P. VAN DER WERF \\ Leiden Observatory \\ P.O. Box 9513 \\ NL - 2300 RA Leiden \\ The Netherlands \\ (pvdwerf@strw.leidenuniv.nl)
}

\section{Introduction}

Ultraluminous infrared galaxies (ULIRGs) are major mergers of gas-rich galaxies and are characterized by a molecular interstellar medium that is concentrated in the central kpc, where it forms a dynamically significant, or perhaps dominant, component (Scoville et al., 1991). Sanders et al. (1988) proposed that ULIRGs might harbour, or evolve into, dust-enshrouded quasars, formed in their obscured centers.

\section{Near-infrared $\mathrm{H}_{2}$ emission}

Luminous vibrational $\mathrm{H}_{2}$ emission at $2.12 \mu \mathrm{m}$ is a general feature of ULIRGs. As shown by Van der Werf et al. (1993), the $\mathrm{H}_{2}$ emission in the nearby. ULIRG NGC 6240 peaks between the two remnant nuclei of the merging systems, and is excited by slow shocks in the dense central molecular medium. Recently, we imaged the $\mathrm{H}_{2} v=1 \rightarrow 0 \mathrm{~S}(1)$ emission in the nearby prototypical ULIRG Arp 220 (Fig. 1) and here likewise the $\mathrm{H}_{2}$ emission peaks between the two radio/near-infrared nuclei. Recent high resolution $\mathrm{CO}$ confirm the location of the molecular gas between the two nuclei, as shown by Scoville et al. (1997). These authors argue that the gas in Arp 220 is concentrated in a thin disk, where dissipation gives rise to an inward mass flux of $100-200 M_{\odot} \mathrm{yr}^{-1}$. This dissipation is traced directly by the near-infrared $\mathrm{H}_{2}$ line emission, since the energy is dissipated by the shocks traced by this emission line. The total energy dissipated by the shocks must equal the total energy radiated away, which can be estimated from the observed $\mathrm{H}_{2}$ 


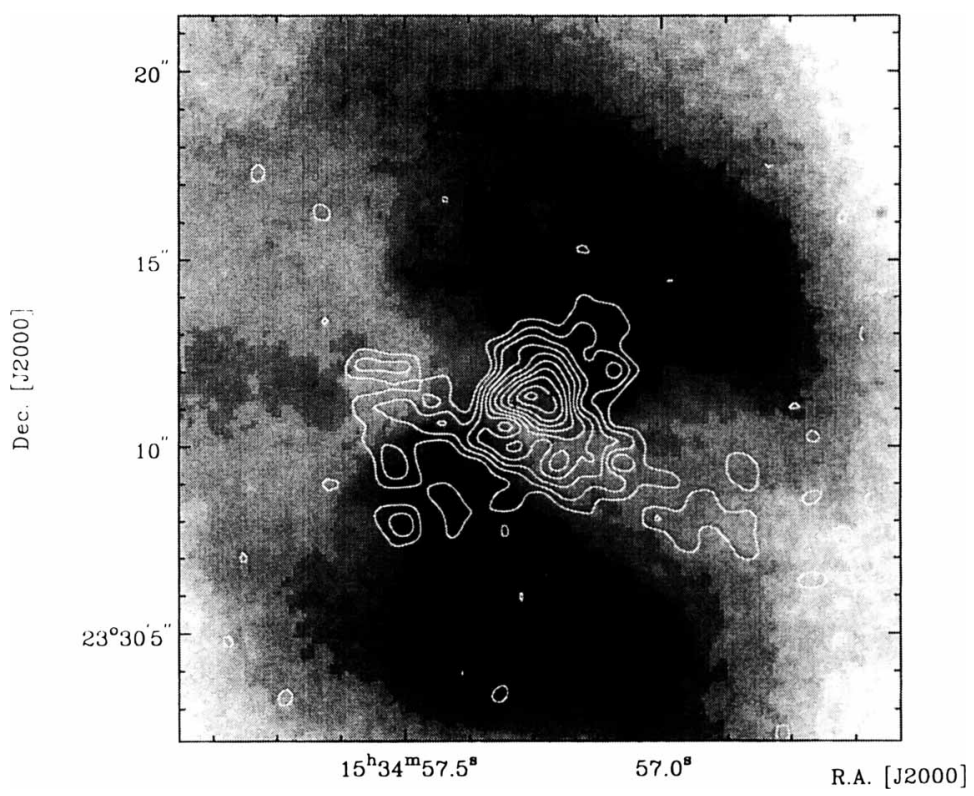

Figure 1. $\mathrm{H}_{2} v=1 \rightarrow 0 \mathrm{~S}(1)$ emission of Arp 220, shown in contours, overlaid on on F555W HST WFPC1 image. The two crosses denote the positions of the radio/near-infrared nuclei (Van der Werf \& Israel, in preparation).

$v=1 \rightarrow 0 \mathrm{~S}(1)$ emission line flux. This argument can be used to estimate an inward mass flux directly from the $\mathrm{H}_{2} v=1 \rightarrow 0 \mathrm{~S}(1)$ emission. The resulting value of $\sim 50 M_{\odot} \mathrm{yr}^{-1}$ is in reasonable agreement with the value derived from $\mathrm{CO}$, given the simplicity of the analysis.

It is tempting to speculate on a possible connection between this large inward mass flux to a position between the stellar nuclei, and the possible formation of a quasar nucleus at this location. On the other hand, the inflowing molecular gas may be consumed entirely by star formation, since the stellar nuclei, although not at the center of the molecular component, are still located within the molecular medium. The star formation rate derived from the far-infrared luminosity of Arp 220 is $\sim 100 M_{\odot} \mathrm{yr}^{-1}$, in remarkable agreement with the estimates of the inward gas mass flux. Higher spatial resolution observations will be able to solve this important issue.

\section{References}

Sanders, D.B., Soifer, B.T., Elias, J.H., Madore, B.F., Matthews, K., Neugebauer, G., \& Scoville, N.Z., 1988, ApJ, 325, 74

Scoville, N.Z., Sargent, A.I., Sanders, D.B., \& Soifer, B.T., 1991, ApJ, 366, L5

Scoville, N.Z., Yun, M.S., \& Bryant, P.M., 1997, ApJ, 484, 702

Van der Werf, P.P., Genzel, R., Krabbe, A., Blietz, M., Lutz, D., Drapatz, S., Ward, M.J., \& Forbes, D.A., 1993, ApJ, 405, 522 